\title{
Evidence for a Millisecond Pulsar in 4U 1636-53 During a Superburst
}

\author{
Tod E. Strohmayer, and Craig. B. Markwardt ${ }^{1}$ \\ Laboratory for High Energy Astrophysics, NASA's Goddard Space Flight Center, Greenbelt, \\ MD 20771; stroh@clarence.gsfc.nasa.gov,craigm@milkyway.gsfc.nasa.gov \\ ${ }^{1}$ also, Dept. of Astronomy, University of Maryland, College Park, MD 20742
}

\begin{abstract}
We report the discovery with the Proportional Counter Array on board the Rossi X-ray Timing Explorer of highly coherent $582 \mathrm{~Hz}$ pulsations during the February 22, 2001 (UT) "superburst" from 4U 1636-53. The pulsations are detected during an $\approx 800 \mathrm{~s}$ interval spanning the flux maximum of the burst. Within this interval the barycentric oscillation frequency increases in a monotonic fashion from 581.89 to $581.93 \mathrm{~Hz}$. The predicted orbital motion of the neutron star during this interval is consistent with such an increase as long as optical maximum corresponds roughly with superior conjunction of V801 Arae, the optical companion to the neutron star in $4 \mathrm{U}$ 1636-53. We show that a range of circular orbits with velocity $90<v_{n s} \sin i<175 \mathrm{~km} \mathrm{~s}^{-1}$ and fractional phase $0.336>\phi_{0}>0.277$ for the neutron star can provide an excellent description of the frequency and phase evolution. The brevity of the observed pulse train with respect to the 3.8 hour orbital period unfortunately does not allow for more precise constraints. The average pulse profile is sinusoidal and the time averaged pulsation amplitude, as inferred from the half amplitude of the sinusoid is $1 \%$, smaller than typical for burst oscillations observed in normal thermonuclear bursts. We do not detect any higher harmonics nor the putative subharmonic near $290 \mathrm{~Hz}$. The 90\% upper limits on signal amplitude at the subharmonic and first harmonic are $0.1 \%$ and $0.06 \%$, respectively. The highly coherent pulsation, with a $Q \equiv \nu_{0} / \Delta \nu>4.5 \times 10^{5}$ provides compelling evidence for a rapidly rotating neutron star in $4 \mathrm{U} 1636-53$, and further supports the connection of burst oscillation frequencies with the spin frequencies of neutron stars. Our results provide further evidence that some millisecond pulsars are spun up via accretion in LMXBs. We also discuss the implications of our orbital velocity constraint for the masses of the components of $4 \mathrm{U} 1636-53$.
\end{abstract}

Subject headings: Binaries: general - Stars: individual (4U 1636-53) - Stars: neutron - Stars: rotation - X-rays: stars - X-rays: bursts 


\section{Introduction}

The low mass X-ray binary (LMXB) 4U 1636-53 is one of approximately 10 neutron star X-ray binaries which have revealed high frequency pulsations during thermonuclear bursts, hereafter "burst oscillations." The $581 \mathrm{~Hz}$ burst oscillations in 4U 1636-53 were discovered with the Rossi X-ray Timing Explorer (RXTE) by Zhang et al. (1997). These oscillations are almost certainly caused by rotational modulation of a nonuniform X-ray emission pattern on the neutron star surface. Near the onset of bursts this pattern likely takes the form of a hot spot (or possibly a pair of hot spots) induced on the neutron star surface by the ignition of nuclear burning (see Strohmayer, Zhang \& Swank 1997). In the cooling part of the burst lightcurve the modulation pattern is less certain, but it may be induced by $r$-modes excited by the thermonuclear flash (see Heyl 2002), or perhaps "extreme weather," in the form of hydrodynamic vortices induced by thermonuclear heating (see Spitkovsky, Levin \& Ushomirsky 2001). In particular, the large modulation amplitudes, high coherence and long term stability of the frequency are consistent with the rotation scenario (see Strohmayer et al. 1998a; Strohmayer \& Markwardt 1999; Muno et al. 2000 and Giles et al. 2002).

There are now three known accreting millisecond pulsars; SAX J1808.4-3658 (Wijnands \& van der Klis 1998; Chakrabarty \& Morgan 1998), XTE J1751-305 (Markwardt \& Swank 2002), and XTE J0929-314 (Remillard, Swank \& Strohmayer 2002). The latter two having been discovered only very recently. Their spin periods are $401 \mathrm{~Hz}, 435 \mathrm{~Hz}$ and $185 \mathrm{~Hz}$, respectively. Several thermonuclear bursts have been observed from SAX J1808.4-3658 with the BeppoSAX Wide Field Cameras (in 't Zand et al. 1998). In the brightest of the three bursts seen from SAX J1808.4-3658 in 't Zand et al. (2001) found evidence for the presence of $401 \mathrm{~Hz}$ burst oscillations, providing additional support to the idea that burst oscillation frequencies are set by the spin frequencies of neutron stars.

Strohmayer et al. (1998b) suggested that observations of burst oscillations in bursts at different binary orbital phases might allow a determination of neutron star orbital velocities by measuring the orbital Doppler shifts in the burst oscillation frequency. Recently, Giles et al. (2002) have studied the distribution of the highest observed, or "asymptotic," oscillation frequencies in a large sample of bursts from $4 \mathrm{U}$ 1636-53. Their sample spans a time baseline of more than four years. They find a high degree of frequency stability over this timespan and attempt to constrain the neutron star orbital velocity using a portion of the observed asymptotic frequency distribution.

Over the last few years a new regime of nuclear burning on neutron stars has been revealed with the observation of very long $(3-5 \mathrm{hr})$ thermonuclear bursts from six LMXBs (see Cornelisse et al. 2000; Strohmayer \& Brown 2002; Wijnands 2001; Kuulkers et al. 2002; Cornelisse et al. 2002). These bursts likely result from unstable burning of the ashes (as 
for example, carbon) of hydrogen and/or helium burning (see Cumming \& Bildsten 2001; Strohmayer \& Brown 2001; Kuulkers 2001). Of the six superburst sources so far only $4 \mathrm{U}$ 1636-53 has produced more than one event. Strohmayer (see Strohmayer \& Brown 2001) and Wijnands (2001) independently identified the superburst from 4U 1636-53 which ocurred on February 22, 2001; the former in pointed RXTE observations, the latter in the RXTE/ASM data. Using the ASM data Wijnands (2001) also identified a similar event from 4U 1636-53 which occurred 4.7 years earlier, thus providing the first constraint on the recurrence time for superbursts. Only two superbursts have been observed in detail with large area, high throughput instrumentation; a superburst from 4U 1820-30 (Strohmayer \& Brown 2002), and the February 22, 2001 event from 4U 1636-53. Both were observed with the Proportional Counter Array (PCA) on board RXTE.

Here we report the discovery of coherent pulsations at $582 \mathrm{~Hz}$ during the February 22, 2001 superburst from $4 \mathrm{U}$ 1636-53. The plan of this paper is as follows. In $\S 1$ we briefly describe the general properties of the superburst and the data available for high time resolution studies. We then discuss our discovery of the $582 \mathrm{~Hz}$ pulsations and describe in detail the time evolution of the pulsation frequency and the expected behavior given the known binary orbital ephemeris for $4 \mathrm{U} 1636-53$. In $\S 3$ we describe our phase coherent timing study and show that orbital modulation of the frequency fits the data extremely well and that the pulsation is coherent. We close in $\S 4$ with a summary and discussion of the implications of our findings. A detailed study of the spectral evolution and energetics of this superburst will be presented in a future publication.

\section{The February 22, 2001 Superburst}

As part of an approved observing program for 4U 1636-53, RXTE observations were being conducted on February 22, 2001 when a several hour long superburst was observed. During these observations data were sampled in two high time resolution modes, both with a time resolution of 1/8192 seconds. The first was an event mode (E_125us_64M_0_1s) with 64 energy channels and the second a "burst catcher" mode (CB_125us_1M_0_249_H) designed to capture high time resolution lightcurves of bursts across the entire PCA bandpass. Due to the duration and intensity of the superburst, the telemetry rate was very high and portions of RXTE's onboard data recorder, which operates as a circular buffer, were filled before they could be downloaded. Unfortunately, this resulted in the loss of some high time resolution data during the superburst. A similar circumstance ocurred when RXTE observed a superburst from 4U 1820-30 (see Strohmayer \& Brown 2002). In spite of these difficulties, two

intervals of high time resolution data were obtained; the first beginning just before the peak 
of the superburst, and the second in the decaying tail. Data covering the entire burst were obtained in the PCA Standard modes. Standard1 mode data provides 1/8 second resolution lightcurves across the entire PCA bandpass, while Standard2 mode provides 129 channel spectra every 16 seconds. These modes use much less telemetry capacity than the high time resolution modes, and are written to a different virtual channel in the data recorder which was not overwritten.

The 2-60 keV lightcurve of the superburst from Standard1 data is shown in Figure 1a with 1 second time resolution. The rise in count rate beginning near 5500 seconds in the figure represents the source coming out of Earth occultation. For several minutes after this the intensity level was about 4,800 counts/sec, significantly higher than the persistent source rate in the previous orbit. This difference is much too high to be accounted for by variations in the PCA background rate and suggests that the burst may have already begun within the Earth occultation gap. The sharp increase in count rate which follows is real and can be seen with $1 / 8$ second resolution in Figure 1b. This "precursor" began at 16:52:12 February 22,2001 (UTC). The precursor has a timescale characteristic of normal (10 - 20 s duration) type I bursts from $4 \mathrm{U}$ 1636-53, except that it shows an interesting double peaked structure. This behavior appears similar to that seen in the superburst from 4U 1820-30, which also showed a normal Type I burst precursor at its onset (see Figure 2 in Strohmayer \& Brown 2002). Further study of these interesting aspects of the superburst will be described in a future publication.

\subsection{Detection of $582 \mathrm{~Hz}$ Pulsations}

The first high time resolution data interval began at $17: 00: 45$ UTC on February 22, 2001 and lasted $\approx 2354$ seconds. This interval, which spans the peak of the superburst, is marked by the first set of vertical dashed lines in Figure 1a. We began our timing study by computing FFT power spectra of this interval. We used 1024 second intervals of the full 2 - 60 keV bandpass event mode data to compute two power spectra, each with a Nyquist frequency of $4096 \mathrm{~Hz}$. We then searched these power spectra for significant signals in the vicinity of $581 \mathrm{~Hz}$. It was clear almost immediately that the power spectrum of the first $1024 \mathrm{~s}$ interval contained a significant signal at $\approx 582 \mathrm{~Hz}$. The power spectrum of this interval, rebinned in frequency space by a factor of 8 , is shown in Figure 2 a and reveals a highly significant peak near $582 \mathrm{~Hz}$. Figure 2b shows an expanded view of the region around $582 \mathrm{~Hz}$, revealing a pair of peaks separated by $\approx 0.03 \mathrm{~Hz}$. The highest peak in this power spectrum has a single trial chance probability of $\approx 1 \times 10^{-21}$, so there is no doubt about the detection. The implied frequency width of $0.03 \mathrm{~Hz}$ indicates a quality factor, $Q \equiv \nu_{0} / \Delta \nu>1.94 \times 10^{4}$, 
which without any additional timing analysis, is about a factor of 5 larger than that seen to date in burst oscillations from normal Type I bursts (see Strohmayer \& Markwardt 1999; Muno et al. 2000). We did not detect the signal in the remainder of the first high time resolution interval. The upper limit to the rms pulsed amplitude was $0.34 \%$. The 2 nd high time resolution interval is marked by the 2 nd pair of vertical dashed lines in Figure 1a. We searched this interval but detected no signal similar to that found in the first interval. The upper limit to the rms pulsed amplitude in this interval was $0.43 \%$.

\subsection{Time Evolution of the Pulsation Frequency}

In order to investigate the time dependence of the pulsation frequency and to determine whether it persists throughout the entire interval we computed a dynamic power spectrum using the $Z_{1}^{2}$ power statistic (see Buccheri 1983; Strohmayer \& Markwardt 1999). However, in anticipation of the need for a precise timing analysis, we first barycentered the data using the standard RXTE software (faxbary) and the JPL DE200 solar system ephemeris (Standish et al. 1992). This analysis revealed that the two peaks in Figure 2 result from two segments of detectable pulsations during each of which the pulsation frequency is observed to increase in a roughly linear fashion. The $800 \mathrm{~s}$ interval which contains these two pulse trains is marked by the vertical dotted lines in Figure 1a. Figure 3 shows a contour map of the dynamic $Z_{1}^{2}$ (see below) spectrum as well as the PCA countrate as a function of time. To produce Figure 3 we used $64 \mathrm{~s}$ intervals to compute $Z_{1}^{2}$ and we started a new interval every $16 \mathrm{~s}$. This figure shows that the pulsations persist for hundreds of seconds, much longer than the $\sim 10$ s pulse trains observed in normal bursts. Moreover, the overall frequency drift spans only about $0.04 \mathrm{~Hz}$, which is very small compared with the few $\mathrm{Hz}$ drifts observed in normal burst oscillations (Strohmayer \& Markwardt 1999; Muno et al. 2000). The magnitude of this drift is similar to what could be produced solely by the Doppler motion of the neutron star in its binary orbit. A simple estimate, just using the observed magnitude of the frequency drift, would require $v_{n s} \sin i / c=\Delta \nu / \nu=6.9 \times 10^{-5}$, which is equivalent to a projected velocity of $21 \mathrm{~km} \mathrm{~s}^{-1}$. This number underestimates the velocity, since we have ignored the rate at which the frequency changes. Nevertheless, it suggests that we may be seeing a coherent pulsation modulated by orbital motion of the neutron star.

\subsection{Expected Frequency Evolution Based on Binary Orbital Ephemeris}

The $3.8 \mathrm{hr}$ orbital period of V801 Arae, the optical companion to the neutron star in

$4 \mathrm{U}$ 1636-53, is well known from optical observations. Recently, Giles et al. (2002) have 
used new optical observations to test and slightly modify the ephemeris determined from all historical optical observations by Augusteijn et al. (1998). Their resulting ephemeris: HJD = $2446667.3179(33) \pm(N \times 0.15804693(16))$, predicts the epoch of maximum optical brightness in units of heliocentric Julian day. Although the formal statistical error in projecting this ephemeris forward to the time of the superburst is of order $4 \times 10^{-4}$, there is an $\approx 0.05$ systematic fractional phase uncertainty which results from the fact that the optical lightcurve does not have any sharp fiducial features and also has often shown night to night variations (see Giles et al. 2002 for a discussion).

We used this ephemeris to predict the orbital phase of the $800 \mathrm{~s}$ interval during the superburst which shows pulsations. Hereafter all phases will be given as fractional phases (ie. ranging from 0 to 1). The interval begins at MJD 51962.710160410, which corresponds to a fractional phase of 0.352 relative to optical maximum (phase zero is optical maximum). One addtional piece of information is required to predict the corresponding frequency evolution, the relationship between the optical and dynamical ephemerides. For systems like $4 \mathrm{U}$ 1636-53, which show sinusoidal optical modulations and which have inclinations $\lesssim 60^{\circ}$ the standard interpretation has been that optical maximum corresponds to superior conjunction of the optical secondary, that is, when the secondary is furthest from the observer. Figure 4 shows the predicted frequency behavior versus orbital phase of a coherent pulsation associated with the neutron star (as, for example, its spin frequency) given this relationship between the optical and dynamical phases. The predicted phase range of the superburst pulsation interval is denoted with vertical solid lines. The predicted frequency evolution is strikingly similar to that observed, moreover, even an uncertainty in the phase of $\approx 0.1$ would not change the qualitative conclusion that the frequency should be increasing because of the orbital motion. The original Augusteijn (1998) ephemeris predicts an orbital phase of 0.26 for the start of the oscillation interval. This is still in the range of orbital phases which would predict an increasing pulsation frequency. These considerations strongly suggest that the observed frequency variations are consistent with the orbital motion of the neutron star.

Since frequency drift is a common feature of normal burst oscillations, it is a fair question to ask whether the frequency variations observed in this superburst — which we claim to be orbitally modulated - could be mimicked by intrinsic drifts unrelated to the orbit. We argue that this possibility is unlikely for a number of reasons. First, an intrinsic drift would need to replicate the apparent orbital modulation at the phase of the observed burst. As we show below, the observed frequency variation is well fit with a concave upwards curvature which is rarely, if ever, seen in normal burst oscillations (see Strohmayer \& Markwardt 1999; Muno et al. 2002). A second consideration is that the frequency drift observed in the $4 \mathrm{U} 1636-$ 536 superburst is much smaller than that of all other normal burst oscillations. Examining bursts from many different X-ray binaries, Muno et al. (2002) found 68 with oscillations, and 
measured the frequency drifts. Of those, about 30 had oscillations which began more than one second from the burst onset. However, none had drifts smaller than $\sim 0.3 \mathrm{~Hz}$. Therefore we put the chance of observing a $0.04 \mathrm{~Hz}$ drift at 1 in 30 , or $\sim 97 \%$ confidence. To derive a more robust estimate would require more knowledge of intrinsic frequency drifts associated with superbursts, however, with only a single example so far, this is not currently feasible.

The frequency drift in the burst oscillation discussed here is consistent with a range of orbital phase, whose size gives a rough estimate of the likelihood of a chance agreement with orbital modulation. This likelihood is related to the accuracy with which the phase can be predicted based on the optical ephemeris and the length of the pulse train. Obviously, the more of the orbit covered by the pulse train, the greater our confidence would be in an orbital modulation interpretation. Based on the $0.05-0.1$ uncertainty in phase noted above and the $\approx 900$ s pulse train, we can estimate that only about a $5 \%$ to $10 \%$ range of orbital phase could produce a signature consistent with the observed drift. Below we fit orbital models and derive a range of phase spanning about $6 \%$ of the orbital phase which can produce an acceptable fit. So, assuming all the observed frequency drift is intrinsic and not orbital there is roughly a $6 \%$ chance that it could be consistent with the expected orbital modulation. In our opinion, however, this should be considered an upper limit, since it seems quite unlikely that all the observed drift is intrinsic.

In summary, the observed frequency drift appears to be well consistent with that expected based on the known orbital parameters of V801 Arae. A chance agreement assuming all the observed drift were intrinsic to the superburst can be ruled out with confidences at the $94-97 \%$ level.

\section{Phase Coherent Timing Analysis}

In order to test the orbital modulation conclusion more quantitatively we performed coherent timing analyses using the complex $Z_{1}$ statistic (see Strohmayer \& Markwardt 1998 for an example of the use of the $Z_{1}^{2}$ statistic in a similar context), where;

$$
Z_{1}=X+i Y=\left(\sum_{j=1}^{N} \cos \phi_{j}\right)+i\left(\sum_{j=1}^{N} \sin \phi_{j}\right)
$$

The $Z_{1}^{2}$ statistic is defined as $Z_{1}^{2}=Z_{1}^{*} Z_{1}$, and the phase angle is given by $\psi=\tan ^{-1}(Y / X)$. Here $\phi_{j}=2 \pi \int_{0}^{t_{j}} \nu\left(t^{\prime}\right) d t$, where $\nu\left(t^{\prime}\right)$ is the frequency as a function of time, and $t_{j}$ are the observed X-ray event times. 


\subsection{Solution Using Total $Z_{1}^{2}$ Power}

We used a circular orbit model to describe the time variation of the pulsation frequency and phase, vis;

$$
\nu(t)=\nu_{0}\left(1-\frac{v_{n s} \sin i}{c} \sin \left(\omega_{\text {orb }} t+\phi_{0}\right)\right) .
$$

Here, $\nu_{0}, v_{n s} \sin i / c, \phi_{0}$ and $\omega_{\text {orb }}$ are the barycentric frequency, the projected neutron star velocity, the orbital phase at the start of the pulsation interval, and the orbital period, respectively. We used this model to predict the pulsation phase at time $t$ and we varied the parameters in order to maximize $Z_{1}^{2}$. We used the data from the $\sim 800 \mathrm{~s}$ segment shown in Figure 3, but we excluded events in an $160 \mathrm{~s}$ segment between the two pulse trains in which we did not detect significant pulsations. We fixed the orbital frequency $\omega_{\text {orb }}$, since it is known with high precision and we cannot hope to constrain it further with such a short data segment, and we let $\nu_{0}, v_{n s} \sin i / c$, and $\phi_{0}$ vary. We began by making a grid search over a range of parameter space which produced, "by eye," reasonably plausible solutions. We then used a general function extremizer, TNMIN (see Nash 1984; implemented in IDL by C. M.), to optimize the solutions. We double checked the maximization using a simplex method (AMOEBA) implemented in FORTRAN. The two methods were in excellent agreement. We find that this model can describe the frequency and phase evolution very well. Our best fits have $Z_{1}^{2} \approx 276$ which represents an enormous improvement compared to a model with no frequency evolution.

As the data segment is short compared to the 3.8 hour orbital period it is possible to find statistically similar solutions with a range of orbital parameters. Indeed, there is a broad ridge in $Z_{1}^{2}$ space on which the three parameters are strongly correlated. The best model in the sense of having the largest $Z_{1}^{2}=275$ has $\nu_{0}=582.1454 \mathrm{~Hz}, v_{n s} \sin i=136 \mathrm{~km} \mathrm{~s}^{-1}$ and $\phi_{0}=0.293$. However, models with $90 \mathrm{~km} \mathrm{~s}^{-1}<v_{n s} \sin i<175 \mathrm{~km} \mathrm{~s}^{-1}$ are not strongly excluded. A look at Figure 4 confirms why this is so. As long as the fitted orbital phase range is such that the curvature is concave up, then the other parameters, $v_{n s} \sin i / c$, and $\nu_{0}$ can more or less adjust to approximate the observed frequency evolution.

Given the systematic uncertainty of order 0.05 in the optical phase and the additional uncertainty of the exact relationship between optical and dynamical phase it is not possible to prefer a priori a particular $\phi_{0}$, however, the range above is consistent with what is expected based on the optical ephemeris as long as optical maximum ocurrs within $\approx 0.1$ of superior conjunction of the secondary. These considerations show that the data can be well explained by a range of reasonable orbits, however, because of the shortness of the pulse train we cannot

place tighter constraints on the orbital parameters using these data alone. Nevertheless the observed pulsations are highly coherent as we are able to track $\approx 500,000$ consecutive cycles. 
In Figure 5 we compare the standard FFT power spectrum with the $Z_{1}^{2}$ power spectrum using our best orbital parameters. The pair of weak broad peaks near $581.91 \mathrm{~Hz}$ are from the standard FFT analysis and are effectively the same as the pair of peaks in Figure 2. The narrow peaks near $582.1 \mathrm{~Hz}$ come from the $Z_{1}^{2}$ spectrum and result from the coherent addition of the two pulse trains. The sidelobe peaks are caused by the need to bridge the gap between the two pulse trains. Figure 6 shows the best orbital frequency model plotted over the dynamic $Z_{1}^{2}$ contours, and demonstrates visually that it tracks the frequency vs time contours very well. The success of the coherent timing analysis provides strong evidence that we are seeing the orbital modulation of coherent pulsations during the superburst.

\subsection{Phase Evolution Analysis}

We can also investigate the problem in the context of phase evolution as a function of time. For a given set of model parameters we can compute the phase, $\phi_{j}$, of each x-ray event. We can then break up the full set of events into a set of $M$ bins and compute the phase $\psi_{k}$ for each bin. The phases are simply given by;

$$
\psi_{k}=\tan ^{-1}\left(\sum_{m} \sin \phi_{m} / \sum_{m} \cos \phi_{m}\right),
$$

where the index $m$ runs over all events in bin $k$. We can then compute $\chi^{2}=\sum_{k=1}^{M}\left(\psi_{k}-\psi_{\text {avg }}\right)^{2} / \sigma_{\psi_{k}}^{2}$, where $\psi_{\text {avg }}$ is the average phase angle computed from all $M$ bins. We can then minimize $\chi^{2}$ to find a best fitting model. For a coherent signal the error $\sigma_{\psi_{k}}$ in the phase angle is given simply by $\left.1 / \sqrt{(} Z_{1}^{2}\right)$, a result which we have confirmed with monte carlo simulations. This procedure is essentially equivalent to the phase connected timing analysis of burst oscillations described by Muno et al. (2000).

To further explore the phase coherence of our orbital solutions we broke up our event data into 24 segments and carried out the $\chi^{2}$ minimization described above. We found that the best orbit model was entirely consistent with the model obtained from maximizing the $Z_{1}^{2}$ statistic. The minimum $\chi^{2}$ was 23.3 , which for 20 degrees of freedom (3 parameters and 1 additional degree of freedom from calculating $\psi_{\text {avg }}$ ) gives a statistically acceptable solution. The resulting phase residuals and errors are shown in Figure 7 in units of milliperiods. The rms level of 46 milliperiods is indicated by the dashed horizontal lines. This is equivalent to $79 \mu \mathrm{s}$. Although the residuals are unstructured in the sense they do not show any large systematic deviations, there is some weak evidence for phase jitter (or timing noise in the parlance of radio pulsar timing) with a scale of about $20-40$ milliperiods on timescales of zhundreds of seconds, particularly during the first pulse train. Jitter of a similar magnitude is also suggested by dynamic power spectral and phase coherent studies of some burst 
oscillations (see for example, Wijnands, Strohmayer \& Franco 2000; Muno, Chakrabarty \& Galloway 2002). To find the $90 \%$ confidence limits on the model parameters we found where $\Delta \chi^{2}=6.25$, which is appropriate for a 3 parameter fit. This gives a $90 \%$ confidence range for each orbit parameter of; $582.0390<\nu_{0}(\mathrm{~Hz})<582.2262,90<v_{n s} \sin i\left(\mathrm{~km} \mathrm{~s}^{-1}\right)<175$, and $0.336>\phi_{0}>0.277$.

We also compared the circular orbital model with a strictly linear increase of the frequency. The linear model achieves only a minimum $\chi^{2}=58.2$, indicating that the circular orbit model performs significantly better. Quantifying the significance of the difference in

$\chi^{2}$ with the F-test shows that the additional parameter using the circular orbit model is significant at the $2.3 \times 10^{-5}$ level. We note that the sense of the improvement is such that frequency models with curvature (such that the frequency vs time curve is concave up) fit much better than linear models.

We next used our best timing solution to phase fold the data and obtain an average pulse profile which we show in Figure 8. The units are counts and we repeat 2 cycles for clarity. The pulse profile is consistent with a sinusoid with a half amplitude of $1 \%$ of the mean number of counts. Having computed a coherent solution we also made a sensitive search for harmonics and subharmonics, but we did not detect any higher harmonics nor the $290 \mathrm{~Hz}$ subharmonic (see Strohmayer \& Markwardt 1999; Miller 1999; Strohmayer 2001). The $90 \%$ confidence upper limits to the signal amplitude at the $290 \mathrm{~Hz}$ subharmonic and $1,160 \mathrm{~Hz}$ 1st harmonic are 0.1 and $0.06 \%$ of the mean count rate (rms), respectively.

\section{Implications and Discussion}

The observation of coherent pulsations during a superburst from 4U 1636-53 provides additional strong evidence of a rapidly rotating neutron star in 4U 1636-53. For example, our observation of $\sim 500,000$ pulsation cycles at $582 \mathrm{~Hz}$ is roughly analogous to observing a standard $\sim 1$ s period radio or X-ray pulsar for $\sim 6$ days. There would seem to be little doubt that such stability can only reflect the spin period of the neutron star. In the remainder of this section we discuss some of the implications of our findings.

\subsection{Implications for Burst Oscillations}

Our coherent timing analysis places a constraint on the barycentric frequency of the coherent pulsation in $4 \mathrm{U} 1636-53$ of $582.0390<\nu_{0}<582.2262 \mathrm{~Hz}$. This range is higher than any of the asymptotic burst oscillation frequencies measured for $4 \mathrm{U} 1636-53$ by Giles 
et al. (2002). Our 90\% upper limit is about $0.4 \mathrm{~Hz}$ above the highest asymptotic oscillation frequency (see Giles et al. 2002), but the limits on the barycentric pulsar frequency are reasonably consistent with the total observed spread around this peak (see Figure 6 in Giles et al. 2002). The fact that the implied pulsar frequency is within $\approx 1 \mathrm{~Hz}$ of all the measured asymptotic frequencies provides further evidence that the frequencies observed during the more numerous, short duration $(\approx 10 \mathrm{~s})$ thermonuclear bursts are indeed set by the rotational frequency of the neutron star. However, since the distribution of all asymptotic burst oscillation periods from $4 \mathrm{U}$ 1636-53 are not strongly correlated with orbital phase there arguably must be an additional source of variation in the asymptotic frequencies of burst oscillations above that caused by orbital motion of the neutron star.

There has been much recent theoretical work to provide an explanation of the few $\mathrm{Hz}$ frequency drifts observed in burst oscillations. For example, Cumming et al. (2002) have reevaluated their earlier (Cumming \& Bildsten 2000) theoretical calculations of spin down in the neutron star surface layers produced by hydrostatic expansion and angular momentum conservation during thermonuclear bursts. They conclude that such expansion alone is probably not capable of accounting for the largest observed frequency drifts. More recently, Spitkovsky, Levin \& Ushomirsky (2002) have investigated the effects of burst heating in the atmosphere and show that this can drive strong zonal flows which can also produce frequency drifts. They suggest that some combination of radial uplift and horizontal flows may explain the observed drift. Heyl (2002) has recently proposed that $r$-modes excited by the thermonuclear burst may be responsible for producing the observed modulation, especially in the decaying tails, and that the motions of these waves relative to the star can produce the observed frequency drifts.

Our results provide further impetus for such work. The discovery of coherent pulsations argues compellingly for the spin interpretation, which can be summarized as follows. The observed modulations are caused by the rotation of some anisotropic emission pattern on the neutron star surface. Near burst onset the pattern is most likely a localized "spot" (or perhaps antipodal spots). At late times in the burst tails it may be produced by an oscillation mode, perhaps the $r$-modes suggested by Heyl (2002), or the hydrodynamic vortices proposed by Spitkovsky, Levin \& Ushomirsky (2002). However, the observed frequency drifts and variations in the asymptotic burst frequencies must be accomodated within a succesful model. It seems most likely that the observed frequency drifts result from the fact that the emission pattern is not fixed in the rotating frame of the neutron star. Oscillation modes, as for example the $r$-modes, are not fixed in the rotating frame, and thermonuclear flame fronts can move across the surface of the star. In addition, thermonuclear heating drives both radial and horizontal motions, both of which may contribute to the $\lesssim 1 \%$ frequency drifts. 


\subsection{What Causes the Flux Modulation?}

The coherent pulsations observed during the superburst have an average amplitude of $1 \%$ of the mean $2-30 \mathrm{keV}$ countrate, smaller than is typical for burst oscillations from normal bursts. This raises the interesting question; what causes the flux modulations during the superburst and how does it relate to that which operates during normal bursts? If a weak coherent modulation were always present with some fixed amplitude, then if the source intensity increased-as during the superburst-the modulation could become detectable. This seems unlikely, however, since there are intervals during the burst at roughly similar intensities which do not show detectable pulsations. More likely, there is some intrinsic change in the modulation amplitude caused by the superburst. There is good evidence that these events result from the release of thermonuclear energy at great depth $\left(\approx 10^{13}\right.$ $\mathrm{g} \mathrm{cm}^{-2}$, see Strohmayer \& Brown 2002; Cumming \& Bildsten 2001). If the energy release and/or transport is anisotropic then a flux asymmetry at the surface could be produced. Alternatively, the burst flux might excite an oscillation mode (as, for example, suggested by Heyl 2002). In either case, if such a process produced a weak $1 \%$ modulation which varied in strength by about a factor of two, then the intervals of strongest modulation would be detectable, but because the spectral power varies as the square of the modulation amplitude, a modest drop in the amplitude would render the modulation undetectable. Further insight into these issues could result from a phase resolved spectral study, which we will pursue in subsequent work.

\subsection{Constraints on the Component Masses}

Our coherent timing analysis places a constraint on the projected neutron star velocity. As mentioned above, a range of orbital velocities, initial phases and pulsar frequencies are allowed by the data. Giles et al. (2002) used the observed distribution of asymptotic burst oscillation frequencies to constrain the neutron star velocity. They obtained a $99 \%$ upper limit on $v_{n s} \sin i=50 \mathrm{~km} \mathrm{~s}^{-1}$ using a subset of 18 bursts which showed a tight clustering in asymptotic frequency. Although our inferred velocity range is outside their limit, the difference may reflect the exclusion of some bursts from the analysis by Giles et al. (2002). Indeed, the overall spread in frequency of all the bursts from $4 \mathrm{U} 1636-53$ is not strongly at odds with the higher neutron star velocity inferred from our timing analysis here (see for example Figure 6 in Giles et al. 2002). This does suggest, however, that it will likely be even more difficult to infer reliable neutron star orbital velocities using normal burst oscillation frequencies than indicated by the Giles et al. (2002) study.

A measurement of the neutron star orbital velocity has implications for the masses of 
the binary components in $4 \mathrm{U}$ 1636-53. Although the system inclination is not precisely known, the lack of both eclipses and dips suggests that it cannot be much greater than $\sim 60^{\circ}$ (see for example, Frank et al. 1987). Figure 9 shows the range of allowable masses for the components, with the secondary mass along the abscissa and the neutron star mass along the ordinate. Velocity contours are shown for the limits calculated from our orbital timing solutions. Contours are drawn for two inclinations, $70^{\circ}$ (dashed) and $60^{\circ}$ (solid). The allowed masses are those enclosed by the velocity contours.

In accreting binaries the mean density of the donor star is constrained if the orbital period is known and the star loses mass via Roche lobe overflow (see for example, Rappaport, Joss \& Webbink 1982). The constraint can be expressed as;

$$
\frac{m_{2}}{M_{\odot}}=5.427 P_{h r}^{-2}\left(\frac{r_{2}}{R_{\odot}}\right)^{3},
$$

where $P_{h r}, m_{2}$ and $r_{2}$ are the orbital period in hours, the mass of the donor, and the radius of the donor, respectively. If one assumes a particular mass - radius relation for the donor star, then the above constraint yields a mass estimate. If one takes the canonical mass - radius relation for main sequence stars (that is, $m_{2} \approx r_{2}$ ), then for $4 \mathrm{U} 1636-53\left(P_{h r}=3.79\right)$ this gives $m_{2}=0.43 M_{\odot}$. If instead one uses an empirical mass - radius relation for zero age main sequence (ZAMS) stars then the mass estimate is reduced somewhat to $0.34<\left(m_{2} / M_{\odot}\right)<$ 0.37 (see Patterson 1984; Smale \& Mukai 1988). Note, however, that the empirical mass radius relation in the range $m_{2}<0.4 M_{\odot}$ is quite poorly defined (see Patterson 1984; Baraffe et al. 1998).

As can be seen in Figure 9 the mass estimates above are more or less consistent with our derived limits, but perhaps uncomfortably so, since they cluster near the lower velocity limit. Kolb, King \& Baraffe (2001) have recently investigated mass estimates in compact binaries. They show that both mass loss and evolution of the donor prior to the onset of mass exchange can bias the mass estimate. They argue that typically the real mass will be less than the estimate obtained from the Roche lobe constraint and an assumed ZAMS mass - radius relation. These effects, if present, would tend to worsen the agreement with our lower range of masses in Figure 9. One can ask what mass - radius relation would yield a mass estimate more comfortably within the constraints in Figure 9. For example, to have a mass of $0.5 M_{\odot}$ the donor's mass - radius relation would need to be $m_{2} \approx r_{2}^{1.15}$. This does not represent a large discrepancy from expectations, particularly given the uncertainties at the low mass end.

The constraints shown in Figure 9 are purely statistical in nature. It seems likely, however, that a systematic uncertainty may also be present because the observed pulse train spanned only about $7 \%$ of the orbit, and there is some indication of phase jitter from our 
timing analysis. These considerations suggests that there could also be a source of systematic uncertainty in the derived orbital parameters. Because of this, and the sizable uncertainties associated with deriving a mass estimate, for example, the assumed mass - radius relation and the orbital inclination, we do not think that our present mass constraints are strongly at odds with theoretical expectations for the nature of the companion star. However, if further observations continue to favor a high mass for the companion, then it could be an indication that some of the observed frequency drift is intrinsic to the superburst and not caused by orbital modulation. 


\section{REFERENCES}

Augusteijn, T., van der Hooft, F., de Jong, J.A., van Kerkwijk, M.H., \& van Paradijs, J., 1998, A\&A, 332, 561

Baraffe, I., Chabrier, G., Allard, F. \& Hauschildt, P. H. 1998, A\&A, 337, 403

Buccheri, R. et al. 1983, A\&A, 128, 245

Chakrabarty, D. \& Morgan, E. H. 1998, Nature, 394, 346

Cornelisse, R., Kuulkers, E., in't Zand, J. J. M., Verbunt, F. \& Heise, J., 2002, A\&A, 382, 174

Cornelisse, R., Heise, J., Kuulkers, E., Verbunt, F., \& in’t Zand, J. J. M. 2000, ApJ, 357, L21

Cumming, A. \& Bildsten, L. 2000, ApJ, 544, 453

Cumming, A. et al. 2001, ApJ, submitted, (astro-ph/0108009)

Frank, J., King, A. R. \& Lasota, J.-P. 1987, A\&A, 178, 137

Giles, A.B., Hill, K.M., Strohmayer, T. E. \& Cummings, N. 2002, ApJ, 568, 222

Heyl, J. S. 2001, astro-ph/0108450

in 't Zand, J. J. M. et al. 1998, A\&A, 331, L25

in 't Zand, J. J. M. et al. 2001, A\&A, 372, 916

Kolb, U., King, A. R. \& Baraffe, I. 2001, MNRAS, 321, 544

Kuulkers, E. 2001, The Astronomers Telegram, \#68, 68, 1

Kuulkers, E. et al. 2002, A\&A, 382, 503

Markwardt, C. B. \& Swank, J. H. 2002, IAUC, 7870

Miller, M. C. 1999, ApJ, 515, L77

Morgan, E. H. \& Chakrabarty, D. 1998, Nature, 394, 346

Muno, M. P. et al. 2002, ApJ, submitted (astro-ph/0204320)

Muno, M. P., Fox, D. W., Morgan, E. H. \& Bildsten, L. 2000, ApJ, 542, 1016

Nash, S. G. 1984, SIAM J. Num. Anal., 21, 770

Patterson, J. 1984, ApJS, 54, 443

Remillard, R. A., Swank, J. H. \& Strohmayer, T. E. 2002, IAUC, 7893

Smale, A. P. \& Mukai, K. 1988, MNRAS, 231, 663

Spitkovsky, A., Levin, Y. \& Ushomirsky, G. 2001, ApJ, 566, 1018 
Standish, E. M., Newhall, X. X., Williams, J. G. \& Yeomans, D. K 1992, in Explanatory Supplememt to the Astronomical Almanac, ed. P. K. Seidelmann (Mill Valley: University Science), 239

Strohmayer, T. E. \& Brown, E. F. 2002, ApJ, 566, 1045

Strohmayer, T. E. 2001, Advances Sp. Res., vol. 28, Nos 2-3, 511

Strohmayer, T.E., Zhang, W., \& Swank, J.H., 1997, ApJ, 487, L77

Strohmayer, T. E., Zhang, W., Swank, J. H., White, N. E. \& Lapidus, I. 1998a, ApJ, 498, L135

Strohmayer, T.E., Zhang, W., Swank, J.H., \& Lapidus, I., 1998b, ApJ, 503, L147

Strohmayer, T.E., \& Markwardt, C.B., 1999, ApJ, 516, L81

Wijnands, R. 2001, ApJ, 554, L59

Wijnands, R., \& van der Klis, M. 1998, Nature, 394, 344

Zhang, W. W., Lapidus, I., Swank, J. H., White, N. E. \& Titarchuk, L. 1997, IAUC, 6541 


\section{Figure Captions}

Fig. 1.- Lightcurve of the superburst from 4U 1636-53 observed on February 22, 2001 (top). The data are the $2-60 \mathrm{keV}$ PCA countrates from the Standard1 datamode. In the top panel the time resolution is $1 \mathrm{~s}$. The dashed vertical lines denote the intervals with high time resolution event mode data. The $\sim 800 \mathrm{~s}$ interval in which pulsations are detected is shown by the vertical dotted lines. An exploded view of the sharp rise near the start of the burst is also shown (bottom). Here the time resolution is $1 / 8 \mathrm{~s}$. Note the double peaked profile which shows timescales typically seen in normal Type I X-ray bursts $(\sim 10 \mathrm{~s})$. Time is measured from 15:19:00 UTC on Feb. 22, 2001.

Fig. 2.- Power spectrum (top) of the first 1024 second data interval starting at 17:00:44 UTC. The spectrum was computed using the full bandpass event mode data. The Nyquist frequency is $4096 \mathrm{~Hz}$. The frequency resolution is 1/128 Hz. The significant peak near 581 $\mathrm{Hz}$ is evident. An expanded view of the region around $582 \mathrm{~Hz}$ is also shown (bottom). The frequency resolution in this plot is $1 / 256 \mathrm{~Hz}$. Two peaks separated by $\sim 0.03 \mathrm{~Hz}$ are clearly resolved.

Fig. 3.- Dynamic $Z_{1}^{2}$ power spectrum of the $800 \mathrm{~s}$ interval containing significant pulsations. The $Z_{1}^{2}$ data were computed using $64 \mathrm{~s}$ data intervals and a new interval was started every 16 s. Contours at 16, 18, 20, 22, 25, 30, 35, 40, 45, 50, 60, 70, 80, and 90 are shown. Two pulse trains are clearly evident, separated by a gap of $\sim 200$ seconds. The PCA countrate (4 detectors) is also shown (see right axes). Time is measured from $17: 02: 25$ UTC on Feb. 22,2001

Fig. 4.- Predicted orbital frequency evolution of a coherent pulsation centered on the neutron star in $4 \mathrm{U} 1636-53$ and the orbital phase range of the 800 second pulsation interval during the superburst. We used the optical ephemeris from Giles et al. (2002) along with the single assumption that optical maximum corresponds to superior conjunction of the optical secondary. The vertical lines denote the phase range of the $800 \mathrm{~s}$ pulsation interval. The curve shows the frequency modulation which would be produced with $v \sin i=62 \mathrm{~km} \mathrm{~s}^{-1}$ for the neutron star. This is not intended to represent a fit to the observed frequency evolution, but rather to simply show the qualitative nature of the predicted frequency evolution. Even with an offset of the phase relationship between optical maximum and superior conjunction of $\sim 0.1$ the ephemeris predicts that a pulsation frequency associated with the neutron star should be increasing.

Fig. 5.- Comparison of the FFT power spectrum which assumes no frequency or phase evolution with the $Z_{1}^{2}$ power spectrum computed using the best orbital frequency evolution model. The weak, broad peaks to the left of the figure are from the standard FFT power 
spectrum, while the sharp peaks to the right result from the phase coherent $Z_{1}^{2}$ power spectrum. The sidelobe peaks in the coherent spectrum result from the gap between the two pulse trains.

Fig. 6.- Dynamic $Z_{1}^{2}$ contours as a function of frequency and time. Also shown is the frequency evolution from the best orbit model. The solid curve shows the model with $\nu_{0}=$ $582.1454 \mathrm{~Hz}, v_{n s} \sin i=136 \mathrm{~km} \mathrm{~s}^{-1}$ and $\phi_{d y n}=0.293$ and has a $Z_{1}^{2}=275.5$. See the caption to Figure 3 for the reference epoch.

Fig. 7.- Pulse phase residuals (observed - model) for our best fitting orbit model. The rms deviation is $\sim 46$ milliperiods and is denoted by the dashed horizontal lines. There is some modest indication of an additional phase jitter in the first pulse train. See the caption to Figure 3 for the reference epoch.

Fig. 8.- Average pulse profile in the full 2 - $60 \mathrm{keV}$ band produced by folding the two observed pulse trains with the best fitting orbit model. The mean number of counts, 447340, has been subtracted. The profile is sinusoidal and has a half amplitude of $1 \%$ of the mean number of counts. Phase zero is arbitrary.

Fig. 9.- Allowable component masses for 4U 1636-53 inferred from the neutron star orbital velocity constraints. A pair of velocity limits are plotted for two representative orbital inclinations, $60^{\circ}$ (solid), and $70^{\circ}$ (dashed). The curves are labelled with their respective velocities. 

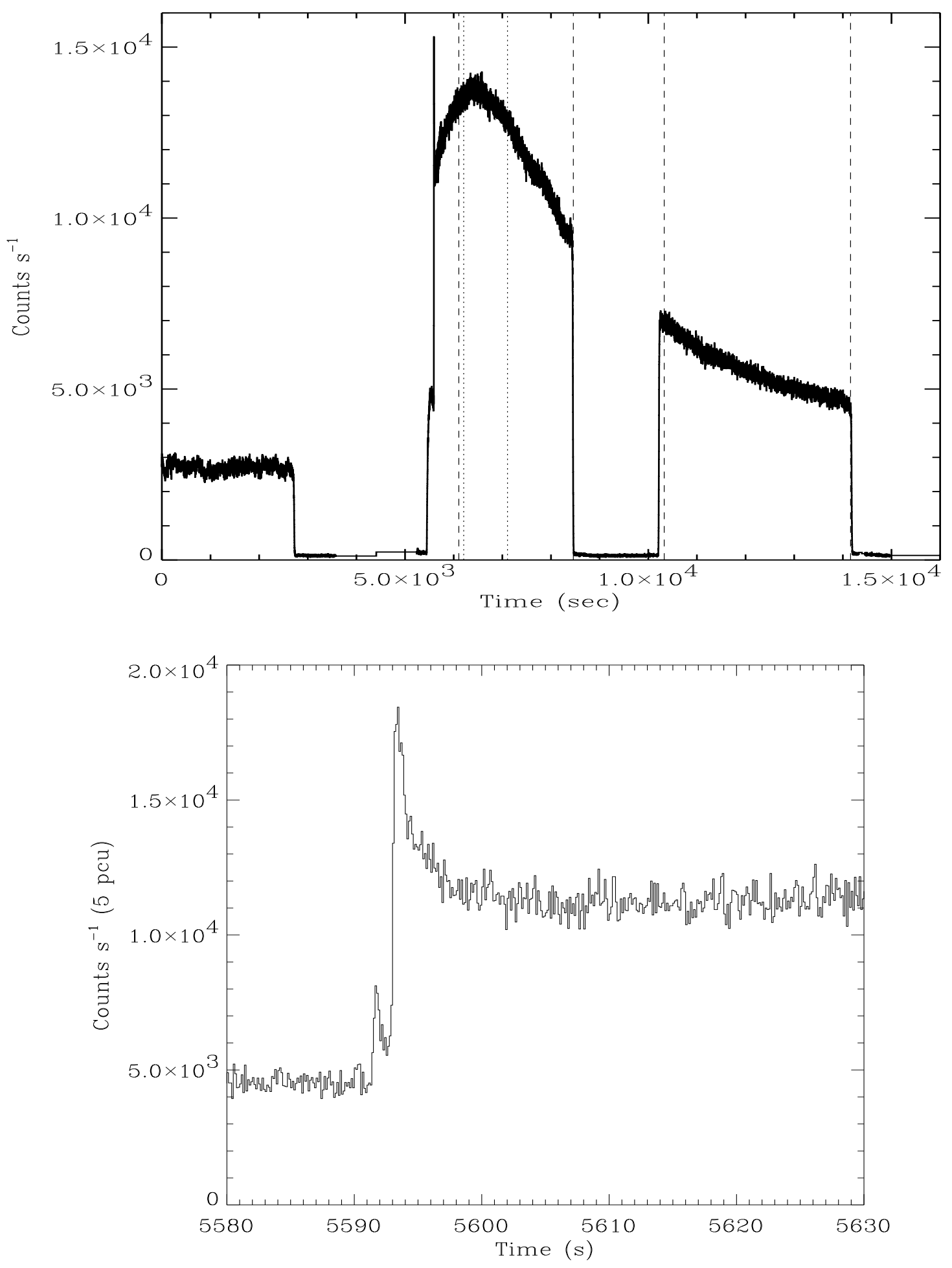

Figure 1: Lightcurve of the superburst from 4U 1636-53 observed on February 22, 2001 (top). The data are the $2-60 \mathrm{keV}$ PCA countrates from the Standard1 datamode. In the top panel the time resolution is $1 \mathrm{~s}$. The dashed vertical lines denote the intervals with high time resolution event mode data. The $\sim 800 \mathrm{~s}$ interval in which pulsations are detected is shown by the vertical dotted lines. An exploded view of the sharp rise near the start of the 

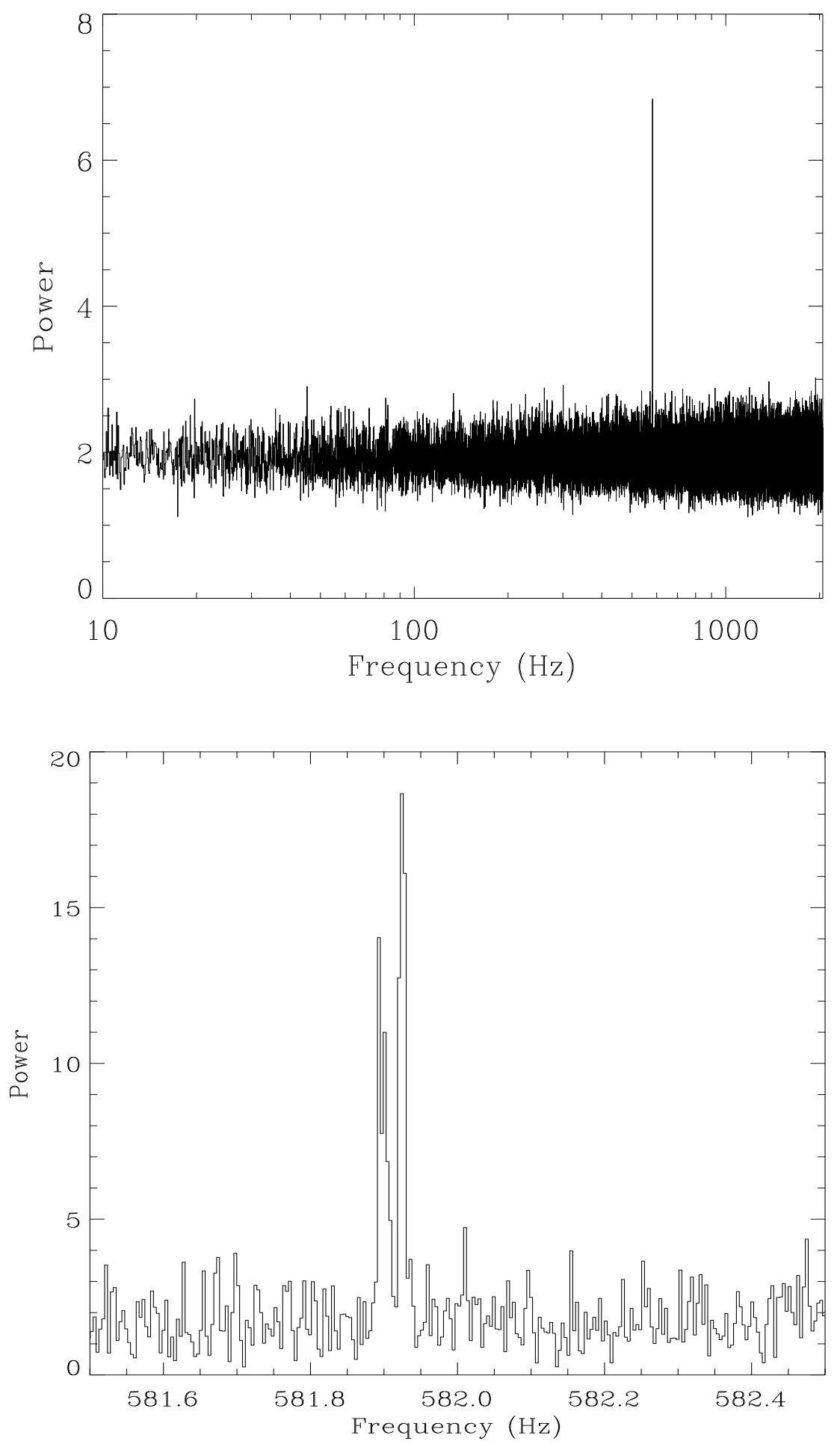

Figure 2: Power spectrum (top) of the first 1024 second data interval starting at 17:00:44 UTC. The spectrum was computed using the full bandpass event mode data. The Nyquist frequency is $4096 \mathrm{~Hz}$. The frequency resolution is $1 / 128 \mathrm{~Hz}$. The significant peak near 581 $\mathrm{Hz}$ is evident. An expanded view of the region around $582 \mathrm{~Hz}$ is also shown (bottom). The frequency resolution in this plot is $1 / 256 \mathrm{~Hz}$. Two peaks separated by $\sim 0.03 \mathrm{~Hz}$ are clearly resolved 


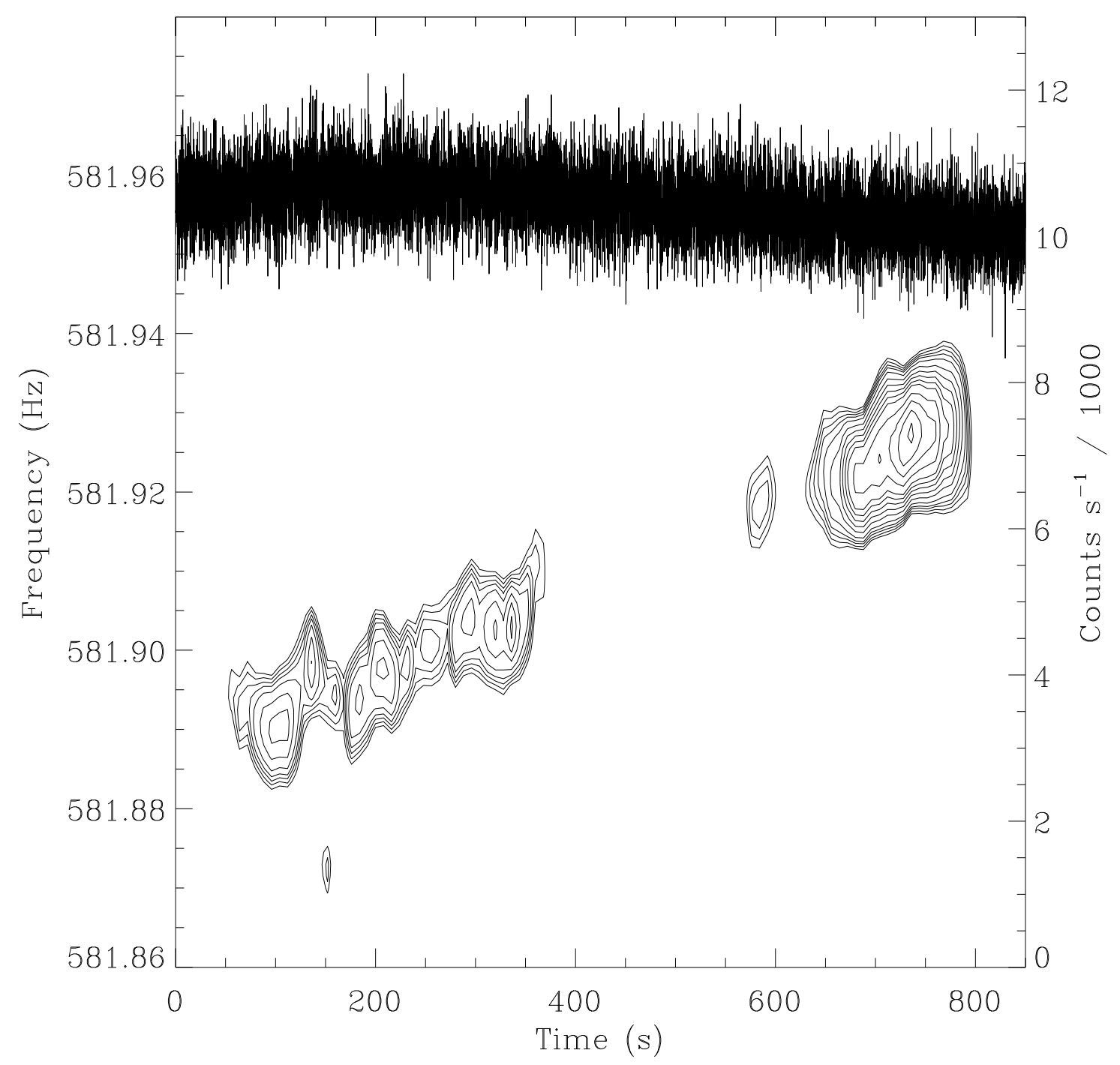

Figure 3: Dynamic $Z_{1}^{2}$ power spectrum of the $800 \mathrm{~s}$ interval containing significant pulsations. The $Z_{1}^{2}$ data were computed using $64 \mathrm{~s}$ data intervals and a new interval was started every 16 s. Contours at 16, 18, 20, 22, 25, 30, 35, 40, 45, 50, 60, 70, 80, and 90 are shown. Two pulse trains are clearly evident, separated by a gap of $\sim 200$ seconds. The PCA countrate (4 detectors) is also shown (see right axes). Time is measured from $17: 02: 25$ UTC on Feb. $22,2001$. 


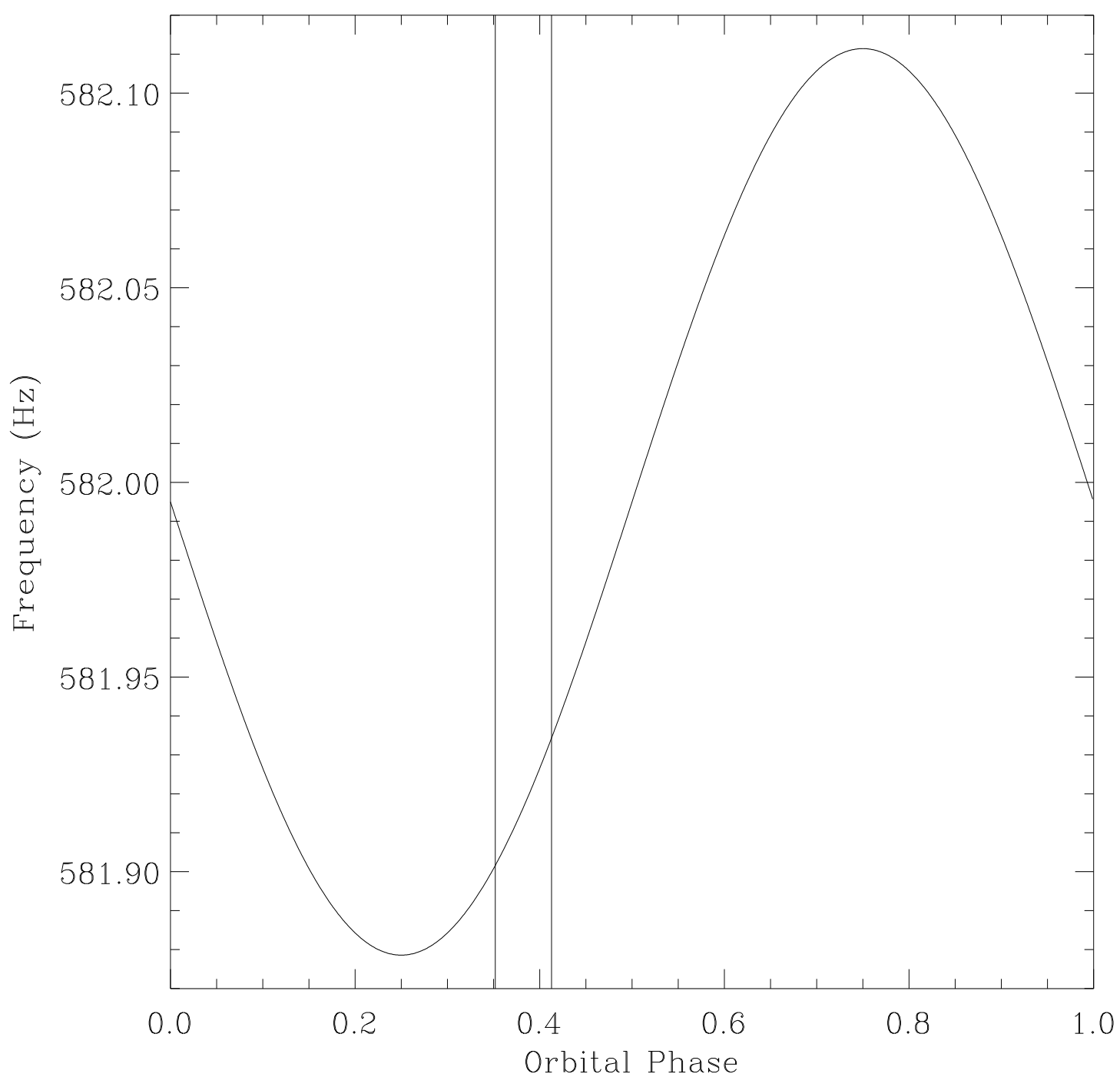

Figure 4: Predicted orbital frequency evolution of a coherent pulsation centered on the neutron star in $4 \mathrm{U}$ 1636-53 and the orbital phase range of the 800 second pulsation interval during the superburst. We used the optical ephemeris from Giles et al. (2002) along with the single assumption that optical maximum corresponds to superior conjunction of the optical secondary. The vertical lines denote the phase range of the $800 \mathrm{~s}$ pulsation interval. The curve shows the frequency modulation which would be produced with $v \sin i=62 \mathrm{~km} \mathrm{~s}^{-1}$ for the neutron star. This is not intended to represent a fit to the observed frequency evolution, but rather to simply show the qualitative nature of the predicted frequency evolution. Even with an offset of the phase relationship between optical max and superior conjunction of $\sim 0.1$ the ephemeris predicts that a pulsation frequency associated with the neutron star should be increasing. 


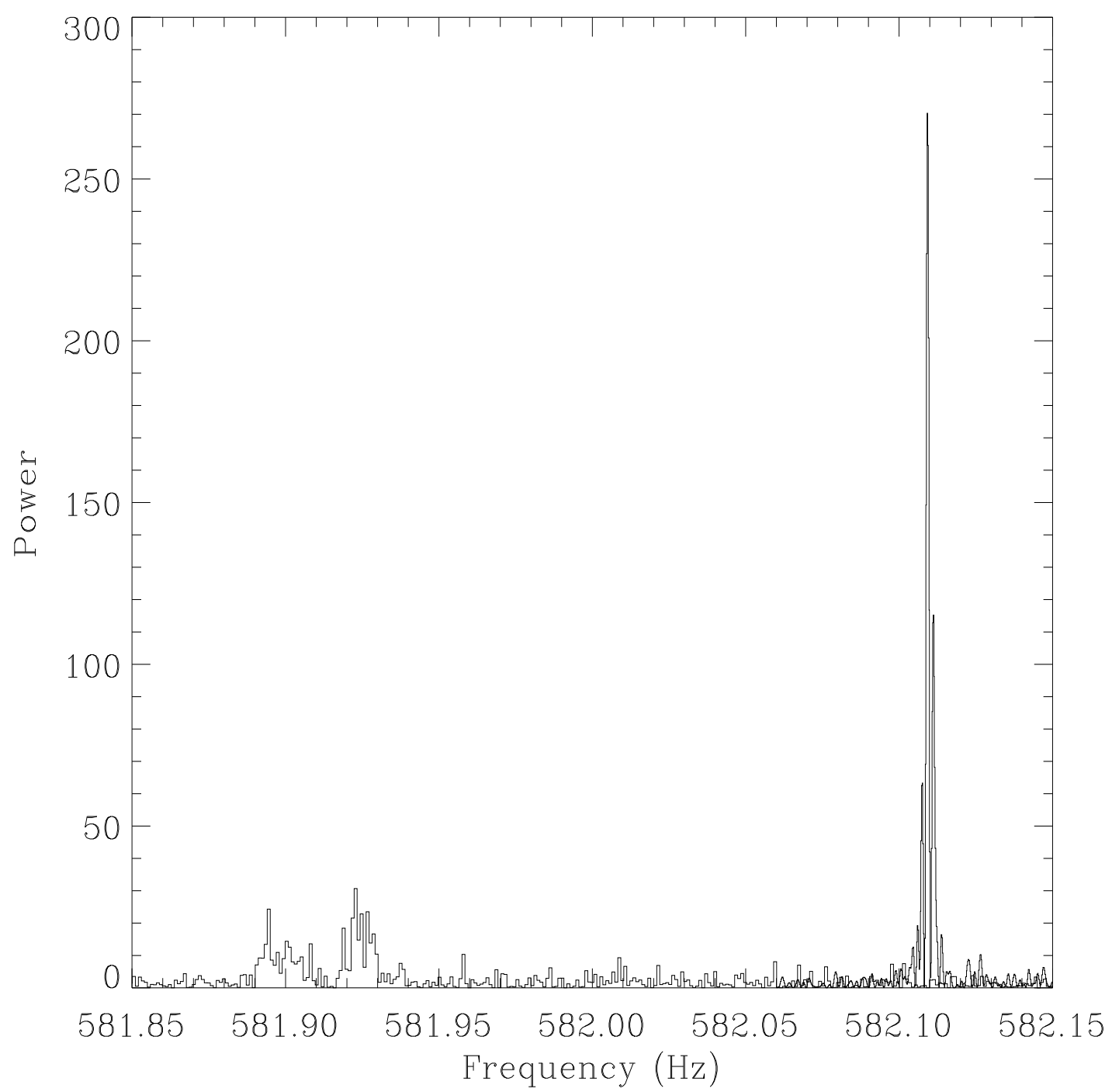

Figure 5: Comparison of the FFT power spectrum which assumes no frequency or phase evolution with the $Z_{1}^{2}$ power spectrum computed using the best orbital frequency evolution model. The weak, broad peaks to the left of the figure are from the standard FFT power spectrum, while the sharp peaks to the right result from the phase coherent $Z_{1}^{2}$ power spectrum. The sidelobe peaks in the coherent spectrum result from the gap between the two pulse trains. 


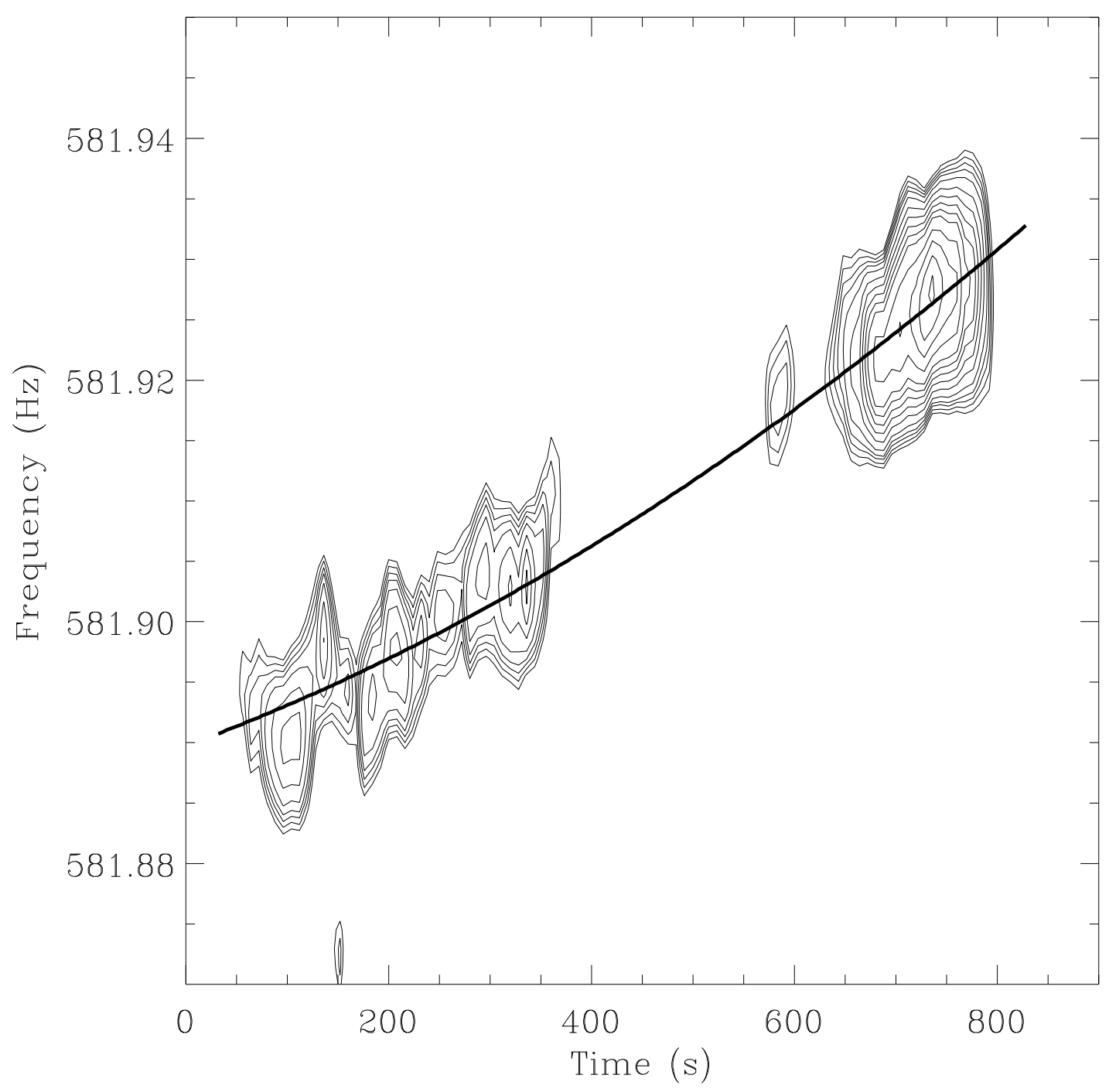

Figure 6: Dynamic $Z_{1}^{2}$ contours as a function of frequency and time. Also shown is the frequency evolution from the best orbit model. The solid curve shows the model with $\nu_{0}=$ $582.1454 \mathrm{~Hz}, v_{n s} \sin i=136 \mathrm{~km} \mathrm{~s}^{-1}$ and $\phi_{d y n}=0.293$ and has a $Z_{1}^{2}=275.5$. See the caption to Figure 3 for the reference epoch. 


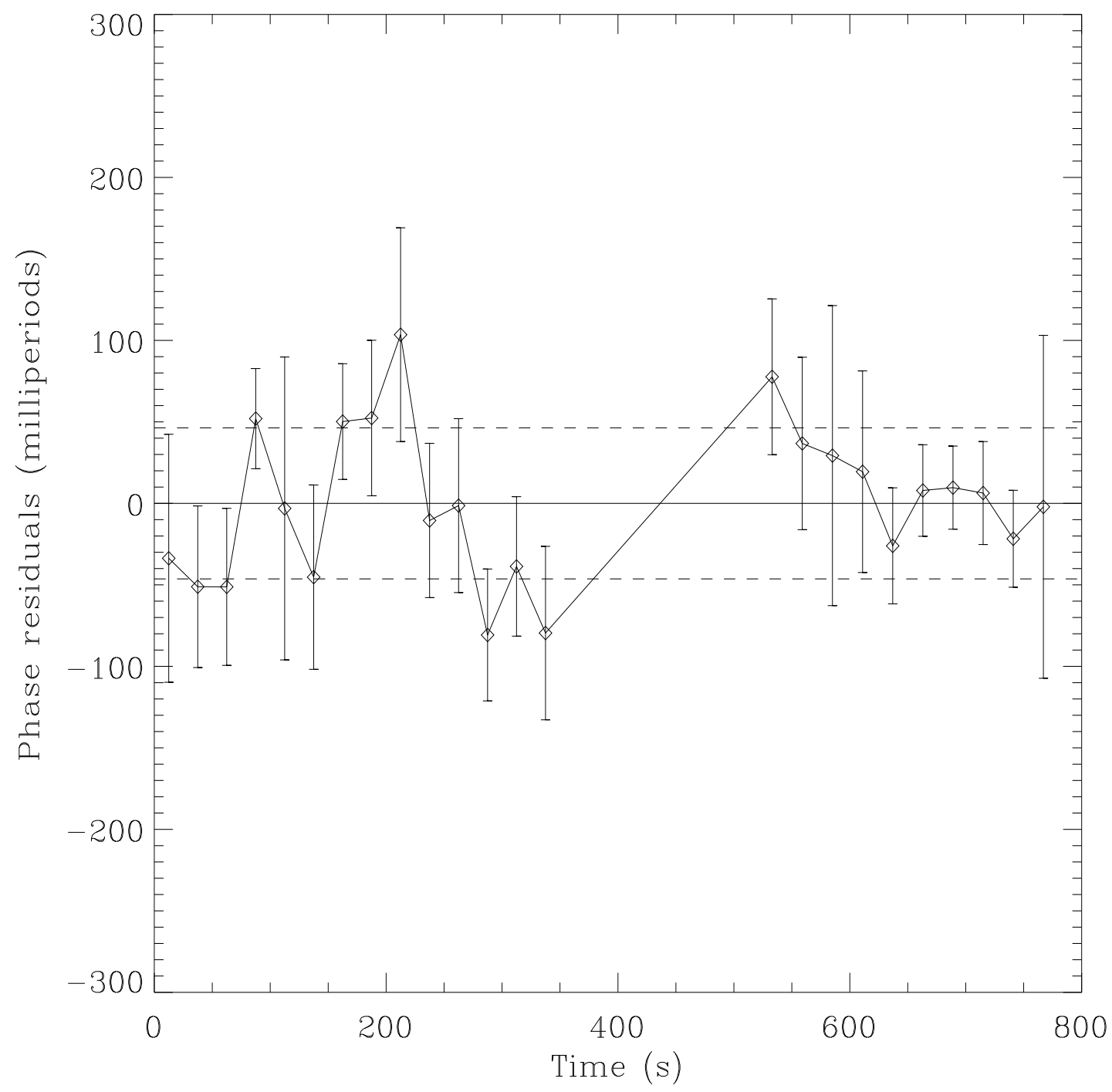

Figure 7: Pulse phase residuals (observed - model) for our best fitting orbit model. The rms deviation is $\sim 46$ milliperiods and is denoted by the dashed horizontal lines. There is some modest indication of an additional phase jitter in the first pulse train. See the caption to Figure 3 for the reference epoch. 


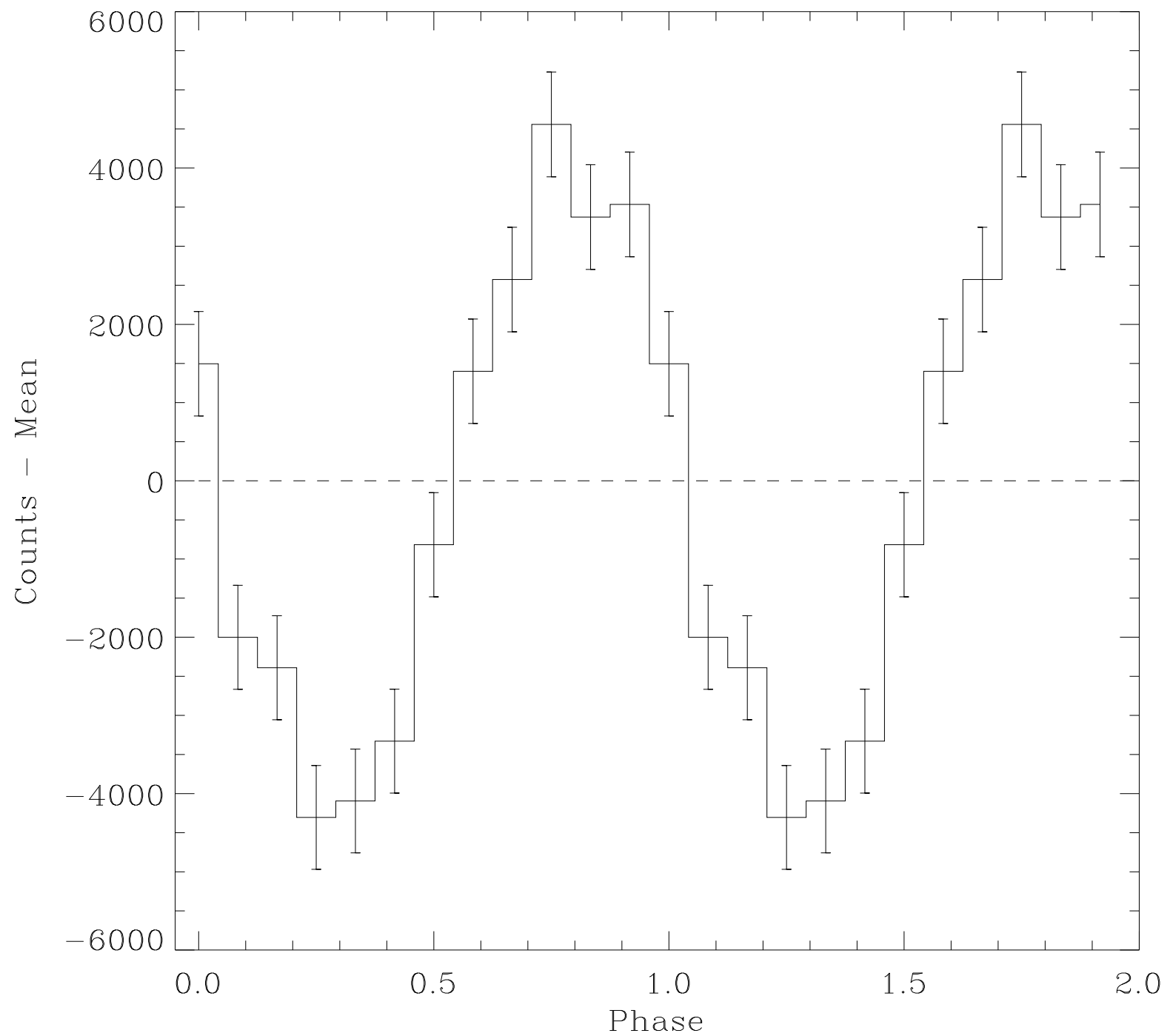

Figure 8: Average pulse profile in the full $2-60 \mathrm{keV}$ band produced by folding the two observed pulse trains with the best fitting orbit model. The mean number of counts, 447340, has been subtracted. The profile is sinusoidal and has a half amplitude of $1 \%$ of the mean number of counts. Phase zero is arbitrary. 


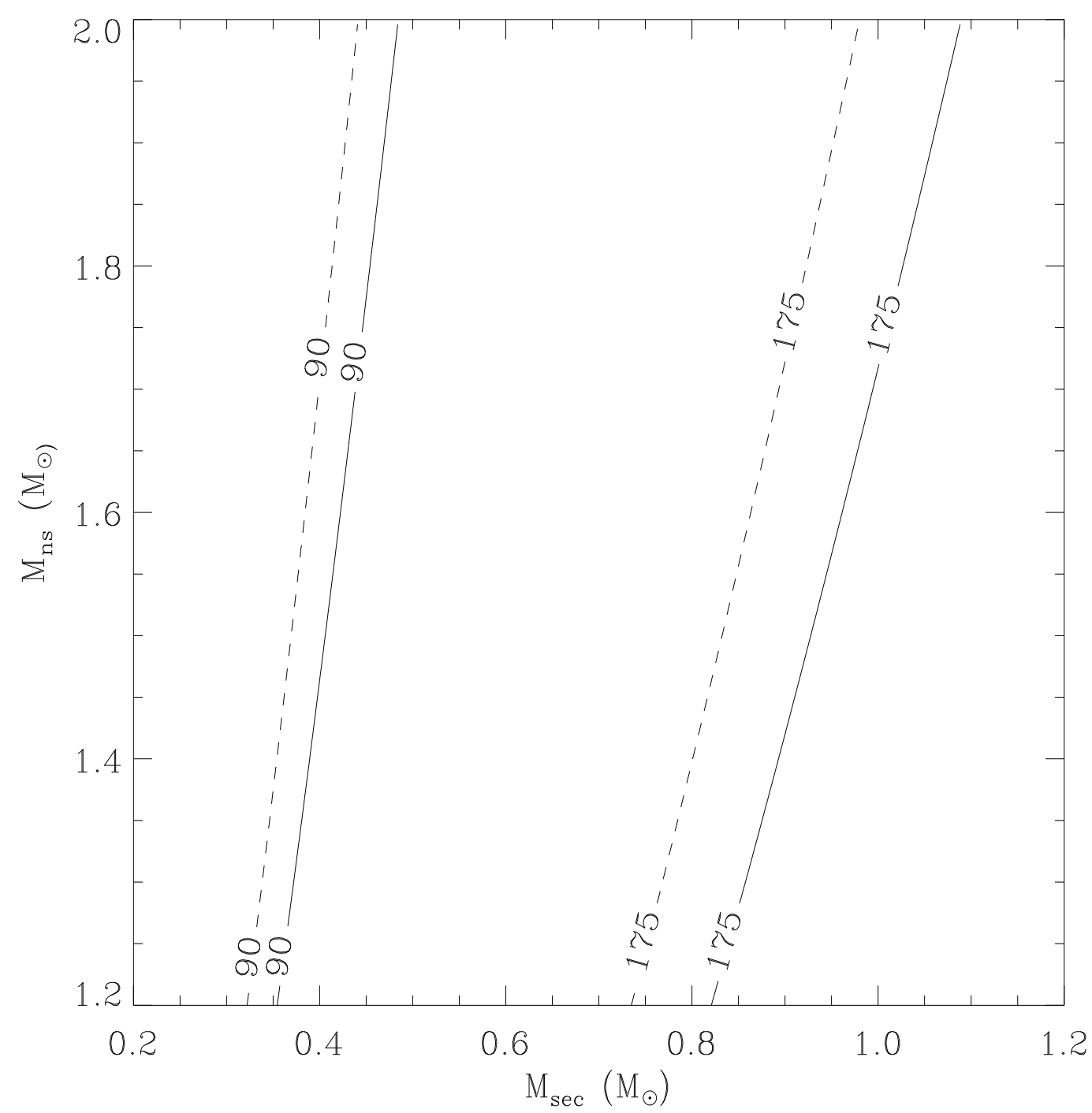

Figure 9: Allowable component masses for 4U 1636-53 inferred from the neutron star orbital velocity constraints. A pair of velocity limits are plotted for two representative orbital inclinations, $60^{\circ}$ (solid), and $70^{\circ}$ (dashed). The curves are labelled with their respective velocities. 